\title{
Two papillary renal cell carcinomas of different origin following renal transplantation (Case report)
}

\author{
HANS-ULRICH GERTH $^{1 *}$, MICHELE POHLEN $^{2 *}$, NILS-HEINRICH THOENNISSEN ${ }^{2}$, BARBARA SUWELACK $^{1}$, \\ HERMANN-JOSEF PAVENSTÄDT ${ }^{1}$, STEFAN STÖRKEL ${ }^{3}$, MAHMOUD ABBAS ${ }^{4}$, \\ TILMANN SPIEKER ${ }^{4}$ and GEROLD THÖLKING ${ }^{1}$ \\ ${ }^{1}$ Department of Medicine D, Division of General Internal Medicine, Nephrology and Rheumatology; \\ ${ }^{2}$ Department of Medicine A, - Hematology/Oncology, University Hospital of Münster, D-48149 Münster; \\ ${ }^{3}$ Department of Pathology, Helios Hospital, D-42283 Wuppertal; ${ }^{4}$ Department of Pathology, \\ University Hospital Münster, Germany
}

Received December 31, 2011; Accepted March 14, 2012

DOI: 10.3892/ol.2012.704

\begin{abstract}
Papillary renal cell carcinoma (PRCC) is a rare malignant tumor entity compared to common clear cell renal carcinoma. In the present study, we report a patient who was diagnosed with PRCC twice and successfully treated each time following renal transplantation. The first PRCC was located in the left native kidney two years following transplantation, and the second PRCC was diagnosed in the allograft 13 years following transplantation. The two tumors were completely removed by surgery in stage I of the disease with sufficient conservation of the allograft function. Notably, the tumors had a different origin as indicated by the microsatellite analysis, which reflects the exceptional course of the case. Risk factors for PRCC were identified in our patient. We concluded that high-risk candidates for malignancies in renal transplant recipients should receive shorter ultrasonic screening intervals, which may facilitate early tumor detection and improve outcome rates.
\end{abstract}

\section{Case report}

A 55-year old Caucasian male was admitted to the University Hospital Münster, Germany, due to a solid mass in the renal allograft observed by routine ultrasonography. The patient had a body mass index (BMI) of $35 \mathrm{~kg} / \mathrm{m}^{2}$ and a tobacco consumption of 30 pack-years. Informed consent for this case report

Correspondence to: Dr Hans-Ulrich Gerth, Department of Medicine D, Division of General Internal Medicine, Nephrology and Rheumatology, University of Münster, Albert Schweitzer-Campus 1, D-48149 Münster, Germany

E-mail: ulrich.gerth@ukmuenster.de

*Contributed equally

Key words: nephrectomy, renal cell carcinoma, renal transplantation was obtained from the patient. The study was approved by the local ethics committee.

Examination of the patient's medical history revealed Goodpasture syndrome that had led to end-stage kidney disease 27 years before. Following 14 years of hemodialysis, the patient received a cadaveric renal transplantation, and immunosuppression was started with a triple combination of tacrolimus, mycophenolate mofetil and prednisolone. Two years after transplantation, a solid tumor was incidentally detected in the left native kidney using routine ultrasound. A native computed tomography (CT) scan revealed a well-defined, heterogeneous large mass $(5.8 \times 5.0 \mathrm{~cm})$ originating from the upper pole (Fig. 1A). Due to a suspected renal malignancy, a laparoscopic radical nephrectomy of the left native kidney was performed. Macroscopic inspection of the explanted organ revealed acquired cystic kidney disease (ACKD) and a $5.5 \times 4.5 \times 4.0 \mathrm{~cm}$ large tumor surrounded by a pseudocapsule, which revealed typical characteristics of a low-grade chromophilic PRCC upon histopathological evaluation (Fig. 1B). The tumor was limited to the kidney parenchyma, and immunohistochemistry indicated positivity for $\alpha$-methylacyl-CoA racemase (AMACR), a highly sensitive diagnostic marker of PRCC (1). There was no evidence of metastatic disease detectable by whole-body CT. Furthermore, the function of the renal graft remained satisfactory with a serum creatinine level of $1.5 \mathrm{mg} / \mathrm{dl}$.

A total of 13 years following transplantation, another suspicious inhomogeneous lesion $(5.4 \times 5.7 \times 6.1 \mathrm{~cm})$ was detected at the upper pole of the allograft by routine ultrasonography and was confirmed by magnetic resonance tomography (MRT) imaging (Fig. 2A). Due to the excellent physical condition of the patient and a well-functioning allograft, an organpreserving enucleation technique was selected. The tumor was excised from the kidney by nephron-sparing surgery (NSS). Notably, histolopathological examination again revealed the rare diagnosis of a low-grade PRCC with no invasion of the pseudocapsule. The cutting edges were free of the tumor and histological analysis demonstrated papillary proliferating atypical epithelial cells positive for AMACR (Fig. 2B). 
A

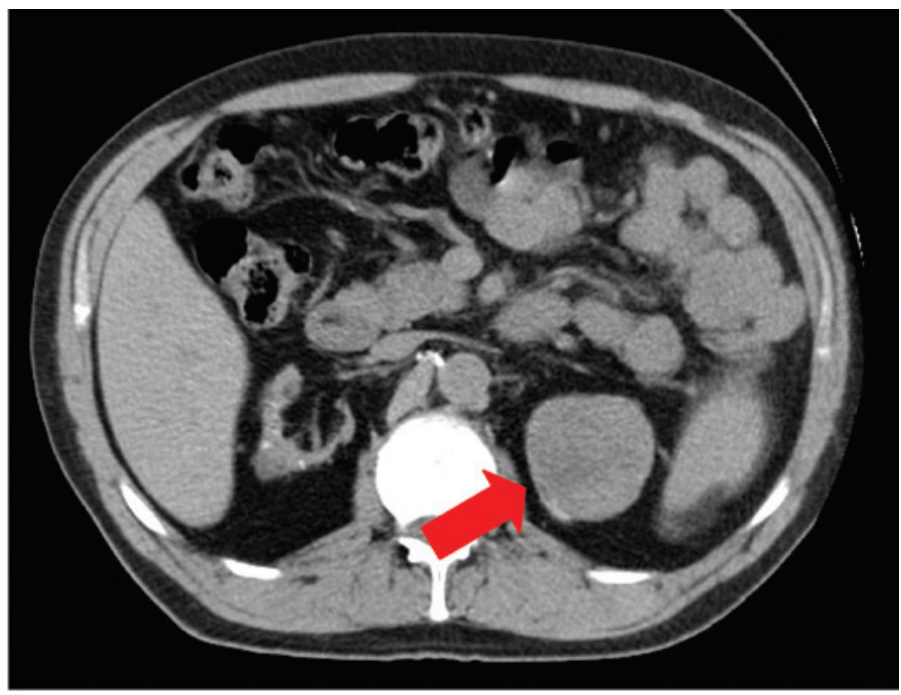

B
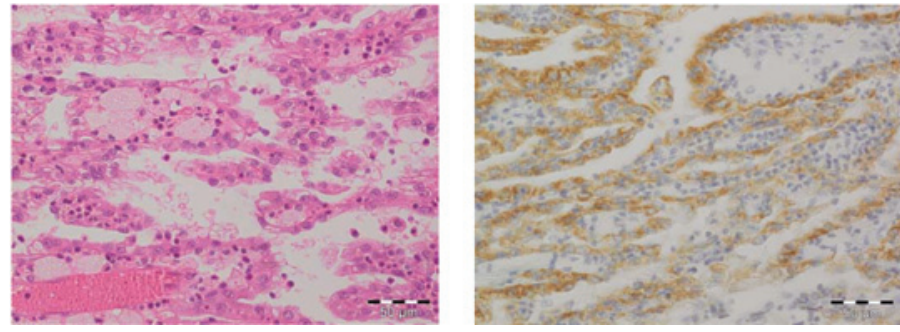

Figure 1. CT scan, histological findings and PCR results of the PRCC in the left native kidney two years following transplantation. (A) A native CT scan revealed a well-defined, heterogeneous large mass $(5.8 \times 5.0 \mathrm{~cm})$ at the upper pole of the left native kidney (arrow); the right kidney demonstrated considerable shrinkage with cysts, typical for ACKD. (B) Histological findings of the first PRCC are shown. The left panel shows the initial low-grade chromophilic PRCC of the left native kidney (H\&E; original magnification, $\mathrm{x} 40$ ); the right panel shows the respective immunohistochemistry with positivity for $\alpha$-methylacyl-CoA racemase (AMACR; original magnification, x40). CT, computed tomography; PRCC, papillary renal cell carcinoma; ACKD, acquired cystic kidney disease.

A

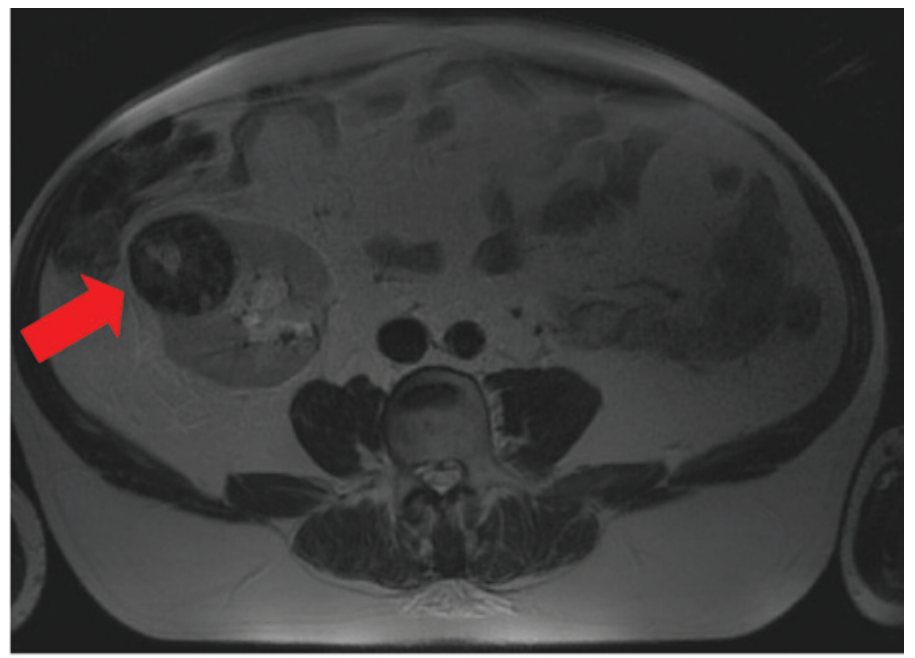

B
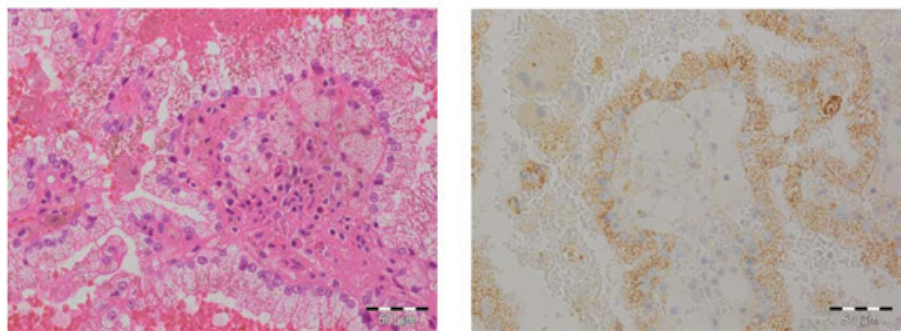

Figure 2. MRT-scan, histological findings and PCR results of the de novo PRCC in the allograft 13 years following transplantation. (A) MRT scan revealed a suspicious inhomogeneous lesion $(5.4 \times 5.7 \times 6.1 \mathrm{~cm})$ at the upper pole of the allograft (arrow). (B) Histological findings of the second PRCC are shown. The left panel shows the de novo low-grade PRCC of the allograft; the proliferating atypical epithelial cells were positive for AMACR (right panel); original magnification, $\mathrm{x} 40$ for the left and right panels. MRT, magnetic resonance tomography; PRCC, papillary renal cell carcinoma; AMACR; $\alpha$-methylacyl-CoA racemase. 
A postoperative whole body CT scan and scintigraphy of the skeletal system revealed no signs of metastasis. The graft remained persistent and well-functioning, and tacrolimus was replaced by everolimus to decrease the potential risk of further tumor development. To confirm the origin of the PRCCs, a DNA microsatellite analysis was performed with samples derived from the two kidney tumors. Notably, PCR amplification revealed different patterns in 5 of 7 regions in the microsatellite analysis, which reflects the different origin of the two tumors.

\section{Discussion}

The overall prevalence of post-transplantation malignancy is significantly increased following renal transplantation $(2,3)$. A total of $5 \%$ of all malignancies are kidney tumors; twice the amount of that in the general population (4). Kidney transplant recipients carry a 15 -fold risk of renal cell carcinoma following transplantation $(5,6)$. Notably, among these approximately $40 \%$ of all renal cell carcinoma are PRCC compared to a prevalence of only $10-15 \%$ in the general population (7-9).

The present case report demonstrates two relevant features. Firstly, the incidence of two PRCCs of different origins in one patient (one PRCC in the native kidney and one in the allograft), which to the best of our knowledge, has not been previously reported. Secondly, short ultrasonography control intervals in a high-risk patient enable early tumor detection and successful therapy. This demonstrates an enormous clinical relevance.

As PRCC is a rare malignant tumor entity, the occurrence of two PRCCs in the same patient following renal transplantation appears to be an uncommon coincidence. There are several well-known risk factors for the development of renal cell carcinoma, including smoking, obesity, abuse of analgesics and ACKD (10). Furthermore, prolonged high-dose immunosuppression following renal transplantation leads to a higher incidence of malignancies (3).

A number of risk factors may have contributed to PRCC development in the patient. Thirteen years of triple immunosuppressive therapy, obesity with a BMI of $35 \mathrm{~kg} / \mathrm{m}^{2}$, ACKD and 30 pack-years tobacco consumption identified our patient as a high-risk candidate for renal malignancies. Therefore, routine ultrasonography screening examinations for malignancies within the native kidney and the allograft are necessary. Although de novo carcinomas in the renal allograft are extremely rare, certain cases of carcinomas detected in the graft have been reported $(11,12)$. Long follow-up periods should be recommended as malignancies can occur even decades following transplantation.
Early tumor diagnosis is essential for a good outcome. In our patient, a diagnosis at stage I of the disease enabled an organ-preserving surgery with a persistent well-functioning graft. In comparison to radical nephrectomy, NSS has become a safe treatment method for low-stage renal cell carcinomas and enables the preservation of organ function $(13,14)$.

In conclusion, this case demonstrates the relevance of risk factors in the development of PRCC. This strengthens the requirement for a risk adaptive screening strategy for malignancies in the allograft and native kidneys. For high-risk candidates, shorter ultrasonic screening intervals may facilitate early tumor detection and enhance the chance for successful NSS and better outcome rates.

\section{References}

1. Szponar A, Beothe T and Kovacs G: How useful is alphamethylacyl-CoA racemase (AMACR) immunohistochemistry in the differential diagnosis of kidney cancers? Histopathology 56: 263-265, 2010.

2. Birkeland SA, Løkkegaard H and Storm HH: Cancer risk in patients on dialysis and after renal transplantation. Lancet 355: 1886-1887, 2000

3. Morath C, Mueller M, Goldschmidt H, Schwenger V, Opelz G and Zeier M: Malignancy in renal transplantation. J Am Soc Nephrol 15: 1582-1588, 2004.

4. Penn I: Primary kidney tumors before and after renal transplantation. Transplantation 59: 480-485, 1995.

5. Kasiske BL, Snyder JJ, Gilbertson DT and Wang C: Cancer after kidney transplantation in the United States. Am J Transplant 4: 905-913, 2004.

6. Suson KD, Sausville JE, Sener A and Phelan MW: Native nephrectomy for renal cell carcinoma in transplant recipients. Transplantation 91: 1376-1379, 2011.

7. Schwarz A, Vatandaslar S, Merkel S and Haller H: Renal cell carcinoma in transplant recipients with acquired cystic kidney disease. Clin J Am Soc Nephrol 2: 750-756, 2007.

8. Ishikawa N, Tanabe K, Tokumoto T, et al: Renal cell carcinoma of native kidneys in renal transplant recipients. Transplant Proc 30: 3156-3158, 1998.

9. Hoshida Y, Tsukuma H, Yasunaga Y, et al: Cancer risk after renal transplantation in Japan. Int J Cancer 71: 517-520, 1997.

10. Alexander MP, Farag YM, Mittal BV, Rennke HG, Tullius SG and Singh AK: De novo multifocal renal cell carcinoma in the renal allograft. Kidney Int 75: 111-114, 2009.

11. Tsaur I, Obermuller N, Jonas D, et al: De novo renal cell carcinoma of native and graft kidneys in renal transplant recipients. BJU Int 108: 229-234, 2011.

12. Roupret M, Peraldi MN, Thaunat O, et al: Renal cell carcinoma of the grafted kidney: how to improve screening and graft tracking. Transplantation 77: 146-148, 2004.

13. Van Poppel H, Da Pozzo L, Albrecht W, et al: A prospective, randomised EORTC intergroup phase 3 study comparing the oncologic outcome of elective nephron-sparing surgery and radical nephrectomy for low-stage renal cell carcinoma. Eur Urol 59: 543-552, 2011.

14. Roos FC, Brenner W, Müller M, et al: Oncologic long-term outcome of elective nephron-sparing surgery versus radical nephrectomy in patients with renal cell carcinoma Stage pT1b or greater in a matched-pair cohort. Urology 77: 803-808, 2011. 